\title{
Properties of Generalised Lattice Ordered Groups
}

\author{
Parimi Radha Krishna Kishore and Dawit Cherinet Kifetew
}

\begin{abstract}
A partially ordered group (po-group) is said to be a generalised lattice ordered group (gl-group) if the underlying poset is a generalised lattice. This paper is a study of some properties of finite subsets of a generalised lattice ordered group (gl-group). Finally obtained a lattice ordered group (l-group) from the given interally closed gl-group and concluded that every integrally closed gl-group is distributive.
\end{abstract}

Index Terms-Poset, lattice, po-group, l-group.

\section{INTRODUCTION}

$\mathbf{M}$ URTY and Swamy [1] introduced the concept of a generalised lattice and Kishore [2], [3], developed the theory of generalised lattices. The theory of lattice ordered groups (l-groups) is well known from the books [4], [5], [6]. The concept of generalised lattice ordered groups (gl-group) introduced and developed by Kishore [7], [8], [9]. This paper is a study of some properties of finite subsets of a gl-group. In this paper, Section II contains preliminaries which are taken from the references [7], [8]. In Section III, we proved some properties of a gl-group with respect to the elements of the glgroup. Section IV discussed some properties of finite subsets of gl-groups. In Section V, we obtained an l-group from a given integrally closed gl-group and finally concluded that every integrally closed gl-group is distributive.

\section{Preliminaries}

The definitions of partially ordered group (po-group), totally ordered group (o-group), lattice ordered group (1-group), directed group are well known from the books [4], [5], [6]. The additive identity element of a po-group is denoted by $0 . G^{+}=\{x \in G \mid x \geq 0\}$ which is called positive cone of a po-group $G$. A po-group $G$ is said to be integrally closed if for any $a, b \in G ; n a \leq b$ for all $n \in \mathbb{N}$ implies $a \leq 0$. A po-group $G$ is said to be semiclosed if for any $x \in G, n \in \mathbb{N} ; n x \geq 0$ implies $x \geq 0$.

The concepts of generalised lattice, subgeneralised lattice, distributive poset are known from [2], [3], [1]. For any finite subset $A$ of a poset $P$, define $L(A)=\{x \in P \mid x \leq a$ for all $a \in A\}$, then the set $\mathcal{L}(P)=\{L(A) \mid A$ is a finite subset of $P\}$ is a semi lattice under the set inclusion. If a poset $P$ is a generalised lattice then $(\mathcal{L}(P), \subseteq)$ is a lattice. A generalised lattice $P$ is distributive if and only if $\mathcal{L}(P)$ is distributive. The dual concepts are also true for $U(A)$ and $\mathcal{U}(P)$.

Definition 1 ([7]): A system $(G,+\leq)$ is called a $\mathrm{gl}$ group (generalised lattice ordered group) if (i) $(G, \leq)$ is a generalised lattice, (ii) $(G,+)$ is a group and (iii) every group

The authors were with the Department of Mathematics, Arba Minch University, P.O.Box 21, Arba Minch, Ethiopia e-mail: parimirkk@gmail.com, cherudawit@yahoo.com.

Manuscript received September 23, 2020; accepted January 16, 2021. translation $x \rightarrow a+x+b$ on $G$ is isotone, i.e., $x \leq y$ implies $a+x+b \leq a+y+b$ for all $a, b \in G$.

Here onwards through out this paper $G$ denotes a gl-group unless specified otherwise. Let $X, Y, A$ and $B$ be subsets of $G$. Define $X \leq Y$ if $x \leq y$ for all $x \in X, y \in Y$. Define $A+X=\{a+x \mid a \in A, x \in X\}$. In particular if $A=\{a\}$ then $A+X=a+X$. Observe that the following conditions are equivalent: (iii) of Definition 1, (iii) ' $: X \leq Y$ implies $a+X+b \leq a+Y+b$ for all $a, b \in G$ and (iii) " $: X \leq Y$ implies $A+X+B \leq A+Y+B$.

Theorem 1 ([7]): For any $x, y, a, b \in G$, we have the following properties: (iv) $a+m u\{x, y\}+b=m u\{a+x+$ $b, a+y+b\}, a+M L\{x, y\}+b=M L\{a+x+b, a+y+b\}$. (v) $M L\{a, b\}=\{0\}$ and $M L\{a, c\}=\{0\} \Longrightarrow M L\{a, b+c\}=$ $\{0\}, m u\{a, b\}=\{0\}$ and $m u\{a, c\}=\{0\} \Longrightarrow m u\{a, b+$ $c\}=\{0\}$. (vi) $M L\{x, y\}=-m u\{-x,-y\}, m u\{x, y\}=$ $-M L\{-x,-y\}$. (vii) $a-m u\{x, y\}+b=M L\{a-x+b, a-$ $y+b\}, a-M L\{x, y\}+b=\operatorname{mu}\{a-x+b, a-y+b\}$. (viii) $x-m u\{x, y\}+y=M L\{x, y\}, x-M L\{x, y\}+y=$ $m u\{x, y\}$.

Definition 2 ([8]): For any $x \in G$, define $|x|=$ $m u\{x,-x\}, x^{+}=m u\{x, 0\}$ and $x^{-}=m u\{-x, 0\}$.

Note that $x^{-}$may also be defined as $x^{-}=M L\{x, 0\}$, but both differ only in negative sign of a set, that is one is negative set of the other. In this paper, we consider as given in Definition 2.

Theorem 2 ([8]): For any $x \in G$, we have (ix) $x^{+}=x+$ $x^{-}, x^{-}=-x+x^{+},(-x)^{+}=x^{-},(-x)^{-}=x^{+}$. (x) If $G$ is semiclosed then $|x|=|-x| \geq 0,|x|=\{0\} \Leftrightarrow x=$ $0, L(|x|)=L\left(x^{+}\right) \vee L\left(x^{-}\right)$. (xi) If $G$ is distributive and semiclosed then $L\left(x^{+}\right) \cap L\left(x^{-}\right)=L(0)$.

\section{Properties OF A GL-GRoup W.R.T. ITS ELEMENTS}

In this section, we prove some properties of a gl-group with respect to elements of the gl-group.

Theorem 3: If $G$ is distributive and semiclosed then for any $x, y \in G$, we have $M L((x-M L\{x, y\}) \cup(y-M L\{x, y\}))=$ $\{0\}$.

Proof: Consider $L((x-M L\{x, y\}) \cup(y-M L\{x, y\}))=$ $L(m u\{0, x-y\} \cup m u\{y-x, 0\})=(L(0) \vee L(x-y)) \cap(L(y-$ $x) \vee L(0))=L(0) \vee(L(x-y) \cap L(y-x))$ (since $\mathcal{L}(G)$ is distributive $)=L(0)$ (since $G$ is semiclosed).

Theorem 4: For any $x, y \in G$, we have $M L(|x|) \leq y$ if and only if $-y \leq x \leq y$.

Proof: Suppose $M L(|x|) \leq y$. Then $L(x) \vee$ $L(-x)=L(m u\{x,-x\})=L(|x|) \subseteq L(y)$ and therefore $L(x), L(-x) \subseteq L(y)$; that is $-y \leq x \leq y$. Conversely, suppose $-y \leq x \leq y$. Then $y \in U(s)$ for some $s \in m u\{x,-x\}$ and therefore $M L(|x|) \subseteq L(|x|) \subseteq L(s) \subseteq L(y)$; that is $M L(|x|) \leq y$. 
Theorem 5: If $G$ is distributive and semiclosed, then for any $x, y \in G$, the following statements are equivalent: (i) $M L\{x, y\}=\{0\}$ (ii) $m u\{x, y\}=\{x+y\}$ (iii) $(x-y)^{+}=$ $\{x\}$ and $(x-y)^{-}=\{y\}$.

Proof: (i) if and only if (ii) is clear by the Theorem 1. Now to prove (iii) if and only if (i): Suppose (iii). Then since $G$ is distributive and semiclosed, we have $L(\{x, y\})=L((x-$ $\left.y)^{+}\right) \cap L\left((x-y)^{-}\right)=L(0)$; that is $M L\{x, y\}=\{0\}$. The converse is clear by the Theorem 1 .

Theorem 6: If $G^{+}$is a subgeneralised meet semilattice of $G$, then for any $x, y, z \in G^{+}$, we have (i) $x \leq y+z$ implies $x=y_{1}+z_{1}$ for some $0 \leq z_{1} \leq z$ and $0 \leq y_{1} \leq y$. (ii) $M L\{x, y+z\} \leq m u(M L\{x, y\}+M L\{x, z\})$.

Proof: (i) Suppose $x \leq y+z$. Then $z \in U\left(z_{1}\right)$ for some $z_{1} \in m u\{0,-y+x\}=-M L\{x, y\}+x$. Therefore, $0 \leq z_{1} \leq$ $z$ and $x=y_{1}+z_{1}$ for some $y_{1} \in M L\{x, y\}$. Since $G^{+}$is a subgeneralised meet semilattice of $G$, we have $M L\{x, y\} \subseteq$ $G^{+}$; this gives $0 \leq y_{1} \leq y$. (ii) Let $s \in M L\{x, y+z\}$. Then by (i) $s=y_{1}+z_{1}$ for some $0 \leq z_{1} \leq z$ and $0 \leq y_{1} \leq y$. Since $y_{1}, z_{1} \leq s \leq x$; we can get $y_{1} \leq y_{2}$ and $z_{1} \leq z_{2}$ for some $y_{2} \in M L\{x, y\}, z_{2} \in M L\{x, z\}$. Therefore, $U(M L\{x, y\}+$ $M L\{x, z\}) \subseteq U\left(y_{2}+z_{2}\right) \subseteq U(s)$ for all $s \in M L\{x, y+z\}$, this implies $U(M L\{x, y\}+M L\{x, z\}) \subseteq U(M L\{x, y+z\})$ and hence the result.

Corollary 1: If $G^{+}$be a subgeneralised lattice of $G$, then for any $x, y, z \in G^{+}$we have $M L\{x, y\}=M L\{x, z\}=\{0\}$ implies $M L\{x, y+z\}=\{0\}$.

\section{PROPERTIES OF A GL-GROUP W.R.T. ITS FINITE} SUBSETS

In this section, we prove some properties of a gl-group with respect to finite subsets of the gl-group.

Theorem 7: For any finite subset $X$ of $G$ and $a, b \in G$, we have the following: (i) $a+m u(X)=m u(a+X), m u(X)+$ $b=m u(X+b), a+m u(X)+b=m u(a+X+b)$ and (ii) $a+M L(X)=M L(a+X), M L(X)+b=M L(X+b), a+$ $M L(X)+b=M L(a+X+b)$.

Proof: For any $s \in m u(X)$, we have $a+x \leq a+s$ for all $x \in X$. Therefore, $a+s \in U(a+X)$ for all $s \in m u(X)$, and this implies $\bigcup_{s \in m u(X)} U(a+s) \subseteq U(a+X)$. On the other hand let $t \in U(a+X)$, then there exists $s \in m u(X)$ such that $-a+t \in U(s)$ and so that $t \in \bigcup_{s \in m u(X)} U(a+s)$. Therefore, $U(a+X) \subseteq \bigcup_{s \in m u(X)} U(a+s)$. Hence, $U(a+$ $X)=\bigcup_{s \in m u(X)} U(a+s)$ and then we get $m u(a+X)=$ $a+m u(X)$. Similarly, we can prove the remaining.

Theorem 8: For any finite subsets $A, B, C$ of $G$, we have the following: (i) $M L(A \cup B)=\{0\}, M L(A \cup C)=\{0\}$ implies $M L(A \cup(B+C))=\{0\}$ and (ii) $m u(A \cup B)=$ $\{0\}, m u(A \cup C)=\{0\}$ implies $m u(A \cup(B+C))=\{0\}$.

Proof: (i) Suppose $M L(A \cup B)=\{0\}, M L(A \cup C)=$ $\{0\}$. Then clearly $0 \in L(A \cup(B+C))$. Now let $p \in L(A \cup$ $(B+C))$, then $p \in L((A+A) \cup(B+A) \cup(A+C) \cup(B+C))=$ $L((A \cup B)+(A \cup C))$, later for any $y \in A \cup C$ we have $p-y \in$ $L(A \cup B)=L(0)$, and this implies $p \in L(A \cup C)=L(0)$. Therefore $M L(A \cup(B+C))=\{0\}$. Similarly, we can prove (ii).

Theorem 9: For any finite subset $X$ of $G$ and $a, b \in G$, we have the following: (i) $M L(X)=-m u(-X)$ (ii) $m u(X)=$
$-M L(-X)$ (iii) $a-m u(X)+b=M L(a-X+b)$ and (iv) $a-M L(X)+b=m u(a-X+b)$.

Theorem 10: Let $G$ be a po-group. Then $G$ is a gl-group if and only if $m u(X \cup\{0\})$ (or $M L(X \cup\{0\})$ ) exists for any finite subset $X$ of $G$.

Proof: Suppose $G$ is a gl-group. Then for any finite subset $X$ of $G$, since $X \cup\{0\}$ is also a finite subset of $G$, we have $m u(X \cup\{0\})$ and $M L(X \cup\{0\})$ are finite subsets of $G$. Conversely, suppose the condition. Let $A$ be a finite subset of $G$. Then for any $x \in A$, by Theorem 7 , we have $-M L(A)=$ $m u(-A)=m u(\{0\} \cup(-A+x))+\{-x\}$ is a finite subset of $G$. Therefore $M L(A)$ is a finite subset of $G$ and clearly $m u(A)$ is a finite subset of $G$.

Theorem 11: Let $G$ be a po-group. Then $G$ is a gl-group if and only if $G^{+}$is subgeneralised join semilattice of $G$ and $G^{+}$generates $G$ (i.e., $G=G^{+}-G^{+}$).

Proof: Suppose $G$ is a gl-group. Then for any finite subset $A$ of $G^{+}$, since $A \subseteq G$ and $A \geq 0$, we have $m u(A) \subseteq G$ and $m u(A) \geq 0$, that is $m u(A) \subseteq G^{+}$. Therefore, $G^{+}$is a subgeneralised join semilattice of $G$. Since every generalised lattice is directed, by theorem 2.1.2(c) of [6] we have $G^{+}$ generates $G$ (i.e., $G=G^{+}-G^{+}$). Conversely, suppose the condition. Let $x \in G$, then $x=a-b$ for some $a, b \in G^{+}$. Observe that an element $g$ is a minimal upper bound of $\{a, b\}$ in $G^{+}$if and only if $g$ is a minimal upper bound of $\{a, b\}$ in $G$. Since $G^{+}$is subgeneralised join semilattice of $G$ and $a, b \in$ $G^{+}$, the set of minimal upper bounds of $\{a, b\}$ in $G^{+}$is a finite subset of $G^{+}$. Then the set of minimal upper bounds of $\{a, b\}$ in $G$ is a finite subset of $G$. Now, $m u\{x, 0\}=m u\{a-b, 0\}=$ $m u\{a, b\}-b$ is a finite subset of $G$. Therefore, $m u\{x, 0\}$ is a finite subset of $G$ for all $x \in G$. Hence, $m u(X \cup\{0\})$ exists for any finite subset $X$ of $G$.

\section{INTEGRALLY CLOSED GL-GROUPS}

In this section, we obtain an l-group from a given integrally closed gl-group and concluded that every integrally closed glgroup is distributive.

Recall from [6] the following results. Let $G$ be a directed po-group and $\mathcal{P}(G)$ be the powerset of $G$. Then observe that the map $\sigma: \mathcal{P}(G) \rightarrow \mathcal{P}(G)$ defined by $\sigma(X)=$ $L U(X)$ is a closure operation on $G$. Moreover for each $X \in \mathcal{P}(G), \sigma(X)=\bigcap_{A \in \mathcal{A}} A$ where $\mathcal{A}=\{A \mid X \subseteq A \subseteq$ $G$ and $\sigma(A)=A\}$.

In the following result, we obtain an 1-monoid (1-group) from a given gl-group (integrally closed gl-group).

Theorem 12: Let $G$ be a gl-group. Then $(\mathcal{L}(G), \oplus, \subseteq)$ is an 1-monoid under the operation $\oplus$ defined by $L(A) \oplus L(B)=$ $L(m u(M L(A)+M L(B)))$ for any $L(A), L(B) \in \mathcal{L}(G)$. Moreover, if $G$ is integrally closed then $\mathcal{L}(G)$ is an l-group.

Proof: Closure: Since $M L(A), M L(B)$ are finite subsets of $G, m u(M L(A)+M L(B))$ is also a finite subset of $G$. Identity: $L(0)$ is the identity element. Associative: Let $L(A), L(B), L(C) \in \mathcal{L}(G)$. Consider $(L(A) \oplus L(B)) \oplus$ $L(C)=L(D) \oplus L(C)$ (where $D=m u(M L(A)+M L(B))$ ) $=L U(X)=X^{*}$ (where $\left.X=M L(D)+M L(C)\right)=$ $\bigcap_{Q \in \mathcal{A}} Q$ where $\mathcal{A}=\left\{Q \mid X \subseteq Q \subseteq G, Q^{*}=Q\right\}$. Consider $L(A) \oplus(L(B) \oplus L(C))=L(A) \oplus L(E)$ (where 
$E=m u(M L(B)+M L(C)))=L U(Y)=Y^{*}$ (where $Y=M L(A)+M L(E))=\bigcap_{P \in \mathcal{B}} P$ where $\mathcal{B}=\{P \mid Y \subseteq$ $\left.P \subseteq G, P^{*}=P\right\}$. To show that $\mathcal{A}=\mathcal{B}:$ Let $Q \in \mathcal{A}$. Then for any $z \in M L(C)$ we have $M L(D)+z \subseteq X \subseteq Q$, this implies $L(D)=L U L(D)=L U(M L(D)) \subseteq(Q-z)^{*}=Q-z$ for all $z \in M L(C)$, later since $M L(A)+M L(B) \subseteq L(D)$ we get $M L(A)+(M L(B)+M L(C))=(M L(A)+M L(B))+$ $M L(C) \subseteq L(D)+M L(C) \subseteq Q$. Then for any $z \in M L(A)$ we have $z+(M L(B)+M L(C)) \subseteq Q$, this implies $L(E)=$ $L U(M L(B)+M L(C)) \subseteq(-z+Q)^{*}=-z+Q$ for all $z \in M L(A)$, later since $Y \subseteq M L(A)+L(E) \subseteq Q$ we have $Q \in \mathcal{B}$. Therefore $\mathcal{A} \subseteq \mathcal{B}$, similarly we can prove $\mathcal{B} \subseteq \mathcal{A}$. Hence $(\mathcal{L}(G), \oplus)$ is a monoid. Translation order preserving: Let $L(A), L(B), L(C) \in \mathcal{L}(G)$ and suppose $L(A) \subseteq L(B)$. Then since $U(M L(B)+M L(C)) \subseteq U(M L(A)+M L(C))$ we have $L(A) \oplus L(C) \subseteq L(B) \oplus L(C)$ and similarly $L(C) \oplus L(A) \subseteq L(C) \oplus L(A)$. Therefore $(\mathcal{L}(G), \oplus, \subseteq)$ is an 1-monoid. Now suppose $G$ is integrally closed. Inverse: Let $L(A) \in \mathcal{L}(G), X=M L(A)$ and $Y=M L(m u(-A))$. Then clearly $L(A) \oplus L(m u(-A))=L U(X+Y) \subseteq L(0)$. On the other hand let $a \in U(X+Y)$. To show that $n a \in U(X+Y)$ for all positive integers $n$ : We prove this by induction on $n$. Assume that it is true for $n=k$, that is $k a \in U(X+Y)$. Then for any $x \in X, y \in Y$ we have $k a \geq x+y$, this implies $y-k a \leq t$ for some $t \in Y$, later since $U(X+Y) \subseteq U(x+t) \subseteq U(x+y-k a)$ we have $a \geq x+y-k a$, that is $(k+1) a \in U(X+Y)$. Therefore the result follows by induction. Now since $G$ is integrally closed we get $0 \leq a$ for all $a \in U(X+Y)$, this implies $L(0) \subseteq L U(X+Y)=L(A) \oplus L(m u(-A))$. Therefore $L(m u(-A))$ is the inverse of $L(A)$. Hence $(\mathcal{L}(G), \oplus, \subseteq)$ is an 1-group.

Recall that every l-group is distributive w.r.t. the lattice operations.

Corollary 2: Every integrally closed gl-group is distributive.

Corollary 3: Every integrally closed gl-group is semiclosed.

Theorem 13: Let $G$ be an integrally closed gl-group. Then for any $x, y \in G$, we have $M L(|x-y|)=$ $M L(m u(M L(m u\{x, y\})-m u(M L\{x, y\})))$. In particular, if $y=0$ then $M L(|x|)=M L\left(m u\left(M L\left(x^{+}\right)+M L\left(x^{-}\right)\right)\right)$.

Proof: Since $L(0) \subseteq L(|x-y|)=L(x-y) \vee L(y-x)$ and by Theorem 12 we have $L(|x-y|)=L(0) \vee L(x-y) \vee$ $L(y-x) \vee L(0)=(L(x) \oplus L(-x)) \vee(L(x) \oplus L(-y)) \vee$ $(L(y) \oplus L(-x)) \vee(L(y) \oplus L(-y))=(L(x) \oplus(L(-x) \vee$ $L(-y))) \vee(L(y) \oplus(L(-x) \vee L(-y)))=(L(x) \vee L(y)) \oplus$ $(L(-x) \vee L(-y))=L(m u\{x, y\}) \oplus L(m u\{-x,-y\})=$ $L(m u(M L(m u\{x, y\})-m u(M L\{x, y\})))$.

\section{ACKNOWLEDGMENT}

The authors would like to thank Professor M. Krishna Murty for the valuable suggestions during the preparation of the paper.

\section{REFERENCES}

[1] M. Murty and U. Swamy, "Distributive partially ordered sets," The Aligarh Bull. of Math, vol. 8, pp. 1-16, 1978.

[2] P. Kishore, M. Murty, V. Sri Ramani, and M. Patnaik, "On generalised lattices." Southeast Asian Bulletin of Mathematics, vol. 33, no. 6, pp. 1091-1104, 2009.
[3] — " "On generalised lattices." Southeast Asian Bulletin of Mathematics, vol. 33, no. 6, pp. 23-26, 2009.

[4] G. Birkhoff, Lattice theory. American Mathematical Soc., 1940, vol. 25.

[5] V. Kopytov and N. Medvedev, The theory of lattice-ordered groups. Springer Science \& Business Media, 2013, vol. 307.

[6] S. Steinberg, Lattice-ordered rings and modules. Springer, 2010.

[7] P. Kishore, "Generalised lattice ordered groups (gl-groups)," International Journal of Algebra, vol. 7, no. 2, pp. 63-68, 2013.

[8] — "Ideals and congruences in a gl-group," International Journal of Algebra, vol. 7, no. 2, pp. 69-72, 2013.

[9] - "Generalised lattice ordered groupoids (gl-groupoids)," International journal of scientific and innovative mathematical research, vol. 2, no. 2, pp. 165-170, 2014. 